\title{
Efecto del ejercicio físico de hidrogimnasia sobre la concentración sérica de inmunoglobulina A en mujeres adultas mayores
}

\author{
Javier Hall-López, Paulina Ochoa-Martínez, Ana M. Miranda Botelho Teixeira, \\ José A. Moncada-Jiménez y Estelio Martin Dantas
}

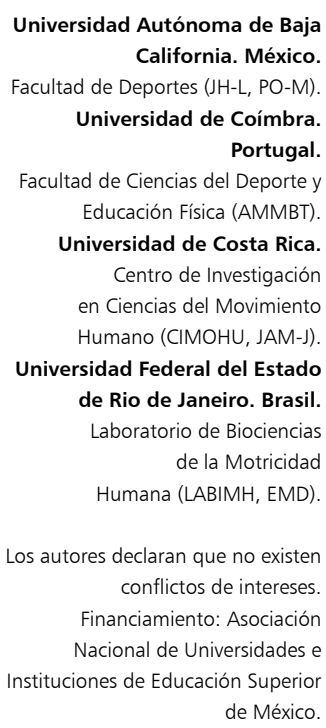

Universidad Autónoma de Baja California. México. Facultad de Deportes (JH-L, PO-M) Universidad de Coímbra. Portugal. Facultad de Ciencias del Deporte y Educación Física (AMMBT).

Universidad de Costa Rica Centro de Investigación en Ciencias del Movimiento Humano (CIMOHU, JAM-J). Universidad Federal del Estado de Rio de Janeiro. Brasil. Laboratorio de Biociencias de la Motricidad Humana (LABIMH, EMD).

Los autores declaran que no existen conflictos de intereses. Financiamiento: Asociación Nacional de Universidades e Instituciones de Educación Superior de México.

Recibido: 6 de junio de 2014 Aceptado: 23 de marzo de 2015

Correspondencia a: Paulina Ochoa-Martínez pochoa@uabc.edu.mx

\section{Effect of hydrogymnastics physical exercise on serum level of immunoglobulin A in elderly women}

Background: Decreases in function of the immune system with age contribute to the increase the risk of infectious diseases especially the upper respiratory tract. On the other hand, physical activity has been widely recommended for health. However, more studies are needed to support the benefit effect of exercise on immune system in elderly. Aim: To evaluate the effect of the hydrogymnastics on the serum level of immunoglobulin A $(\lg \mathrm{A})$ in elderly women. Methods: Twenty-six participants were randomly assigned to an experimental (n: 16) or a control group (n: 10). IgA concentrations were determined by nephelometry (BN2 Analyzer, Dade Behring). The hydrogymnastics training protocol was performed 5 times a week during 12 weeks. The intensity of the aerobic exercise was $50-60 \%$ of the maximum heart rate monitored by telemetry (Polar-FT7). Results: No significant differences (p: 0.797) resulted by ANOVA on serum IgA levels; the percentage of change in experimental group was $\Delta \%=-6.7 \mathrm{mg} / \mathrm{dL}$ compared to the control group, $\Delta \%=-7.4 \mathrm{mg} / \mathrm{dL}$. Conclusion: After three months of hydrogymnastics the IgA level did not show significant change on elderly women; However, positive improvement in percentage of change $\Delta \%$ was found.

Key words: Elderly, exercise, immunoglobulin, health.

Palabras clave: Adultos mayores, ejercicio, inmunoglobulina, salud.

\section{Introducción}

L os mecanismos que hacen funcionar al sistema inmunológico disminuyen con el envejecimiento declinando la producción de linfocitos $\mathrm{T}$ y $\mathrm{B}^{1,2}$; en el sistema óseo al reducirse la cantidad de densidad mineral en el tejido esponjoso se dificulta la hematopoyesis y la migración de linfocitos para su adecuada maduración y función ${ }^{2-4}$. Otro proceso fisiológico que agrava el daño en la producción de linfocitos es el estrés oxidativo que se incrementa con el envejecimiento generando daño en las proteínas de las membranas celulares incluyendo los receptores de las citoquinas, así como daños en el ADN celular de la mitocondria de los leucocitos, disminuyendo así su capacidad de producir energía, causando progresivamente un aumento en la susceptibilidad a enfermedades infecciosas y riesgo de la salud en el adulto mayor ${ }^{5-7}$. Estos procesos biológicos son irreversibles; no obstante, buenos hábitos de salud como una adecuada alimentación, minimizar el estrés psicosocial y el realizar actividad física sistemática pueden ayudar a disminuir el impacto de la inmunosenescencia ${ }^{8}$.

Se ha comprobado que los beneficios del ejercicio en el adulto mayor incrementan la expectativa de vida, se obtiene mejorías en su estado fisiológico y calidad de vida en relación a aquellos adultos mayores sedentarios ${ }^{9,10}$. En el área de estudio de la inmunología y las ciencias aplicadas al ejercicio se han reportado cambios asociados con el paso de la edad que genera más probabilidad de riesgo de contagio de infecciones, principalmente del tracto respiratorio superior ${ }^{11}$; los anticuerpos como la inmunoglobulina A (IgA), protegen contra infecciones y juegan un rol importante de protección en la mucosa respiratoria y gastrointestinal contra virus y bacterias ${ }^{12}$. Por último, estudios refieren menor cantidad de episodios y síntomas de enfermedades infecciosas del tracto respiratorio superior en adultos mayores activos en comparación con sedentarios ${ }^{13}$.

Se ha demostrado en estudios transversales comparativos que adultos mayores que realizan sistemáticamente ejercicio aerobio presentan un menor decline de porcentaje de citoquinas e IgA, que adultos mayores sedentarios ${ }^{14}$. Otros estudios longitudinales en adultos mayores de ambos géneros, divididos en subgrupos de 60 a 69 años de edad y más de 70 años de edad, que realizaron ejercicio aerobio en la modalidad de cicloergómetro por seis meses 
dieron como resultado un aumento significativo de $43 \%$ de IgA salival ( $\operatorname{IgAs})^{15}$. Otros ejercicios, con intensidad baja, en la modalidad de caminata, incrementaron en un tiempo de seis meses las concentraciones de IgA en adultos mayores de 75 años $^{16}$. El ejercicio aerobio en la modalidad de danza, durante 19 semanas en adultos mayores, se tradujo en incrementos significativos en comparación con el grupo control. Otro estudio con el mismo diseño metodológico pero con una duración mayor (32 semanas) demostró aumento significativo en las concentraciones de $\operatorname{Ig} \mathrm{A}^{17}$. Investigaciones donde refieren la combinación de ejercicios de resistencia a la fuerza y actividades aerobias encontraron diferencias significativas respecto al grupo control ${ }^{18}$. Resultados de una investigación han encontrado incrementos de $\operatorname{IgA}$ en adultos mayores que practicaron por 16 semanas de ejercicio en la modalidad de Kouk-Sun-Do ${ }^{19}$.

El efecto del ejercicio de hidrogimnasia sobre las concentraciones séricas de IgA en el adulto es desconocido y se necesitan estudios que avalen los beneficios de esta modalidad en esta población. A partir de los resultados se podrían conocer aspectos que permitan prescribir ejercicios adecuados, su frecuencia, intensidad, duración y sobrecarga en el adulto mayor.

Se realizó la presente investigación al preguntarnos si el ejercicio de hidrogimnasia puede mejorar las concentraciones séricas de IgA en adultas mayores. Objetivo general: evaluar el efecto de un programa de entrenamiento de hidrogimnasia sobre las concentraciones séricas de IgA en adultas mayores, estableciendo una hipótesis sustantiva y otra estadística. La hipótesis substantiva anticipa que se encontrarán diferencias en los niveles séricos de IgA en adultas mayores, participantes en un programa de entrenamiento de hidrogimnasia. La hipótesis estadística se presenta en forma nula y alternativa, teniendo como criterio de aceptación o relación el nivel de $\mathrm{p}<0,05$.

\section{Material y Método}

El presente estudio se consideró de tipo cuasi-experimental, donde la variable independiente fue manipulada para medir su efecto sobre la variable dependiente, con el propósito de determinar el grado de cambio producido por el tratamiento, estableciendo una relación causa-efecto ${ }^{20}$.

La muestra estuvo compuesta por adultas mayores definidas como personas con 60 años de edad o más, conforme a la Organización Mundial de la Salud, voluntarias, que habitan en el municipio de Mexicali, Baja California, México. Según la Comisión Nacional de Población en México, en el 2010 en el Estado de Baja California la proporción de adultos mayores del total de la población era de $6,9 \%$ correspondiendo a 229.914 personas, de los cuales $60 \%$ son mujeres y $40 \%$ hombres. En la ciudad de Mexicali Baja California, la población total de adultos mayores (universo) constituye $64.640^{21}$.

Criterios de inclusión: A partir del universo, la muestra fue seleccionada en forma no probabilística tomando los siguientes criterios de inclusión: tener 60 años de edad o más; ser residente del Municipio de Mexicali, Baja California en México; participar de manera voluntaria en la investigación; no haber participado por lo menos tres meses antes en un programa de ejercicio físico sistemático y ser capaces de realizar las actividades de la vida diaria sin ayuda de terceros.

Criterio de exclusión: Presentar cualquier tipo de condición aguda o crónica que pudiera impedir ejercicios de hidrogimnasia, tales como cardiopatías, diabetes mellitus, hipertensión arterial o asma alérgica no controladas, condiciones músculo esqueléticas que pudieran afectar a la práctica del ejercicio como osteoartritis, lesiones articulares o fracturas recientes, problemas psicológicos y neurológicos y haberse sometido a una cirugía mayor en los últimos seis meses o presencia de enfermedad infecciosa cuyo resultado generara procesos inflamatorios; Por lo tanto la forma se selección del grupo-muestra fue por conveniencia ${ }^{22}$.

La muestra de estudio fue dividida aleatoriamente, por sorteo sencillo, en dos grupos:

- Un grupo control, compuesto por 10 adultas mayores no atendidas con ejercicio de hidrogimnasia.

- Un grupo experimental, compuesto por 16 adultas mayores atendidas con ejercicio de hidrogimnasia.

El presente trabajo atendió al reglamento de la Ley General de Salud en Materia de Investigación para la Salud de México y a los principios éticos de investigación en seres humanos de la declaración de Helsinki ${ }^{23}$.

La entidad en la cual se llevó a cabo esta investigación, realizando las evaluaciones y aplicación del programa piloto en el grupo control y experimental fue el Complejo Acuático Universitario de la Escuela de Deportes de la Universidad Autónoma de Baja California en México. A través de sus directivos se solicitó una carta de información institucional en la que se requirieron las condiciones para realizar la investigación, recibiéndose la anuencia para llevar a cabo el estudio.

Todos los sujetos participantes firmaron una carta de consentimiento informado que consistió en dar a conocer y solicitar al sujeto de estudio el objetivo del proyecto, los procedimientos de evaluación, los posibles riesgos, beneficios y consecuencias, los procedimientos de emergencia y su anuencia de participación de carácter voluntario. El proyecto de investigación fue sometido a evaluación por el Comité de Ética que involucra el estudio con seres humanos, de la Escuela de Deportes de la Universidad Autónoma de Baja California. Protocolo n. 001/2011.

Para la caracterización de la muestra se realizó una 
anamnesis y recolección de datos como edad, sexo, historia de salud y de actividad física. También se midieron los datos antropométricos de masa corporal (MC) y estatura (EST) en conformidad con la International Society for the Advancement of Kinanthropometry (ISAK) obtenidos a través de una balanza digital Tanita ${ }^{\circledR}$ con estadiómetro Seca $^{\circledR}$.

Concentraciones séricas de $\operatorname{IgA}$ : Las muestras de sangre fueron colectadas en la mañana de 7:00 a 8:30 h después de $12 \mathrm{~h}$ de ayuno y $48 \mathrm{~h}$ de reposo de actividad física. Una enfermera calificada recolectó cinco mililitros de sangre venosa colocándolos en tubos EDTA. Las cuantificaciones de $\operatorname{IgA}$ fueron determinadas por nefelómetro (Dade Behring BN2 Nephelometer Analyzer, DADE Behring, USA; utilizando los reactivos de laboratorio IMMAGE Immunochemistry Systems para IgA).

\begin{tabular}{|lcccc|}
\hline \multicolumn{5}{|c|}{ Tabla 1. Estadísticas descriptivas $(\mathrm{M} \pm \mathrm{DE})$ de las mujeres participantes } \\
en el estudio $(\mathbf{n}=\mathbf{2 6})$
\end{tabular}

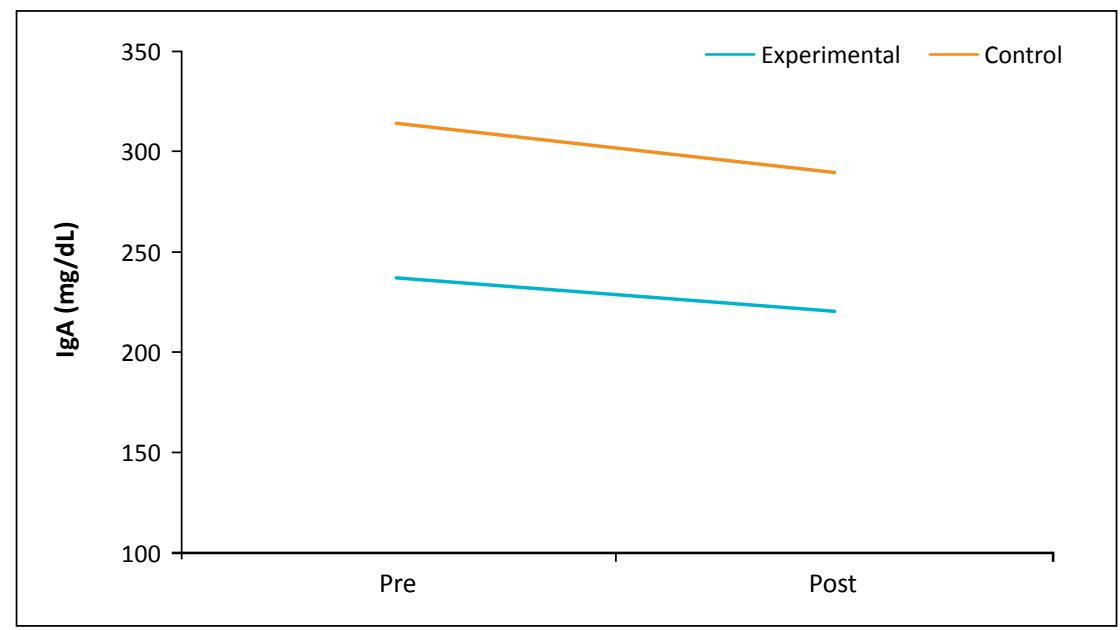

Figura 1. Concentraciones séricas de IgA en mujeres adultas mayores. Nota: el nivel sérico de IgA (inmunoglobulina A) de los sujetos evaluados antes y después del entrenamiento grupo experimental (16) y grupo control (10).
Intervención: A los sujetos participantes se les aplicó un programa de ejercicio físico aerobio de moderada intensidad (en la modalidad de hidrogimnasia) tomando en cuenta las normas generales propuestas por el American College of Sports Medicine (ACSM) ${ }^{24}$, y la Asociación Americana del Corazón (AHA) ${ }^{25}$, consistente en: 60 sesiones, con una frecuencia de cinco veces por semana, con un volumen de 50 min divididos en 10 min de calentamiento, $30 \mathrm{~min}$ de fase medular con intensidad incremental y progresiva de $50 \%$ de la frecuencia cardíaca máxima (FCmáx) las primeras seis semanas y $60 \%$ las últimas seis semanas mediante la siguiente formula FCmáx = 208-0,7x edad ${ }^{26}$, y 10 min de relajación.

Con el empleo de técnicas de estadística descriptiva se buscó caracterizar a la muestra utilizando medidas de localización y de dispersión. Dentro de las primeras estuvieron la media como medida de tendencia central que identifica y localiza el centro del conjunto de los datos; las medias de dispersión estimaron la variabilidad existente en los datos. Con este fin se calculó la desviación estándar (DE), la cual fue utilizada para verificar la simetría de la muestra $^{20}$. Los análisis estadísticos se calcularon con el software EXCEL y SPSS 17.

La segunda parte del tratamiento estadístico corresponde al análisis inferencial del estudio. Para la evaluación de la normalidad de los grupos y la homogeneidad de la varianza de los datos de la muestra, respectivamente, se realizó el test de Shapiro-Wilk. Con el fin del total de posibilidades de comparación inter e intra grupos se realizaron pruebas de análisis de varianza (ANOVA) mixtas $2 \times 2$ (grupos x mediciones) para la variable de concentraciones séricas de IgA.

\section{Resultados}

Participaron en el presente estudio 26 adultas mayores divididas de forma aleatoria en un grupo experimental (GE, n: 16) con una edad de 67,5 \pm 5,4 años, quienes recibieron el programa de entrenamiento de hidrogimnasia y un grupo control (GC, n: 10), con una edad de 67,4 \pm 4,7 años, quienes no tuvieron intervención en el entrenamiento. En la Tabla 1 se observan las características generales de la muestra evaluadas antes y después del entrenamiento de hidrogimnasia.

Se realizaron pruebas de análisis de varianza (ANOVA) mixtas $2 \times 2$ (grupos $x$ mediciones) con un nivel de significancia que se estableció a priori a un $\alpha \leq 0,05$ en las concentraciones séricas de IgA. El ANOVA 2 × 2 de medidas repetidas indicó que no existieron interacciones estadísticamente significativas (p: 0,797) entre los grupos y las mediciones en IgA (Figura 1).

Se encontraron cambios estadísticamente significativos entre los grupos (p: 0,048) pero no entre las mediciones (p: 0,194). 
Se calcularon los porcentajes de cambio $(\Delta \%)$ para cada grupo de estudio mediante el siguiente procedimiento: [(Mediapost-Mediapre)/Mediapre] x 100. Presentándose en el grupo experimental (n: 16$)$ un $\Delta \%=-6,7 \mathrm{mg} / \mathrm{dL}$ en las concentraciones séricas de $\operatorname{IgA}$; en el grupo control (n: 10) se encontró un $\Delta \%=-7,4 \mathrm{mg} / \mathrm{dL}$.

\section{Discusión}

Los resultados del presente estudio abarcan entrenamiento de 60 sesiones de duración, con una frecuencia de cinco veces por semana, durante tres meses. Las concentraciones séricas de IgA no arrojaron resultados estadísticamente significativos a diferencia de estudios previos en adultos mayores donde se aplicaron programas de ejercicios con diferentes modalidades. Tal es el caso de trabajo realizado por Shimizu y cols. ${ }^{15}$, en el que se realizó ejercicio aerobio en la modalidad de cicloergómetro encontrando un aumento significativo (p: 0,05) después de 6 meses de entrenamiento, cinco veces por semana, observándose un cambio por grupo de mujeres de 60-69 años, de $40 \%$, de IgA secretoria-salival (IgAs) ${ }^{15}$, Otra modalidad de ejercicio pero con intensidad baja en la modalidad de caminata incrementó, en un tiempo de seis meses, las concentraciones de IgAs en adultos mayores de 75 años $^{16}$. Otro experimento en adultos mayores demostró que con el ejercicio aerobio en la modalidad de danza, llevado a cabo durante 19 semanas, se produjeron incrementos significativos (p: 0,01) de IgAs, en comparación con el grupo control ${ }^{27}$. Con el mismo diseño metodológico pero con una duración más larga (32 semanas), otro estudio demostró aumentos significativos en los niveles séricos de $\operatorname{IgA}^{17}$. Investigaciones que combinaron ejercicios de resistencia a la fuerza y actividades aerobias también encontraron diferencias significativas (p: 0,01) respecto al grupo control ${ }^{18}$. Otra modalidad de ejercicio donde se han encontrado incrementos séricos de IgA fue en adultos mayores que practicaron por 16 semanas Kouk-Sun-Do ${ }^{19}$.

Para comparar la variable de la IgA con programas de hidrogimnasia se revisó la literatura especializada pero no se encontraron investigaciones que la evaluaran.

Las investigaciones anteriores presentaron resultados positivos en los niveles séricos y salivales de IgA con una duración del entrenamiento entre 4 y 12 meses, aplicando una modalidad de ejercicios cuyo impacto no contrarrestaba el efecto del peso del cuerpo sobre la gravedad, a diferencia de la hidrogimnasia en que el peso del cuerpo puede disminuir hasta en $10 \%{ }^{28,29}$. Al respecto, una posible explicación radicaría en los mecanismos fisiológicos de adaptación derivados del ejercicio, por los cuales, la médula ósea genera hematopoyesis ante el impacto del peso y de esa manera genera y libera leucocitos influyendo en la mejora de las concentraciones séricas de $\operatorname{Ig} \mathrm{A}^{8}$.
Otra diferencia con las investigaciones anteriores fue que el presente estudio estuvo diseñado con una sobrecarga y una intensidad de la frecuencia cardíaca máxima (FCmáx) incremental y progresiva de 50\% (FCmáx) las primeras seis semanas y $60 \%$ las últimas seis semanas.

En conclusión, los estudios anteriores reflejan que se necesita por lo menos 4 a 12 meses para encontrar cambios significativos en las concentraciones séricas y salivales de $\operatorname{Ig} \mathrm{A}^{15,17-19}$.

En términos generales, el presente trabajo de investigación tuvo por objetivo establecer los efectos de un programa de hidrogimnasia sobre las concentraciones séricas de $\operatorname{IgA}$, en adultas mayores. Esto, como propósito de servir de referencia para la planificación, ejecución y evaluación de intervenciones orientadas a prevenir, minimizar y/o eliminar el problema de predisposición a enfermedades infecciosas en los adultos mayores que permita a los investigadores y profesionales en general que trabajan con adulto mayor comprender un funcionamiento más apropiado y tener mayores elementos para proporcionarles una mejor atención. A corto plazo, esta investigación se torna relevante a partir del momento que puede servir de referencia teórica y práctica para implementar estrategias que puedan ser utilizadas en la intervención para adultos mayores.

Como limitaciones del estudio se establece que el grupo control, compuesto por 10 adultas mayores no atendidas con ejercicio de hidrogimnasia, presentó de manera basal un peso corporal e índice de masa corporal y concentraciones séricas de IgA en la evaluación basal mayores que el grupo experimental. Por otra parte, no se cuantificó mediante cuestionario un seguimiento de enfermedades del tracto respiratorio en los sujetos de estudio.

A partir de lo anteriormente expuesto, recomendamos para futuras investigaciones un control mayor de las variables a estudiar como lo son los hábitos de vida, alimentación y actividad física, evitando una posible alteración de los resultados obtenidos. Además, recomendamos un período de observación de los resultados, meses después de finalizar el estudio piloto, y al mismo tiempo, estudiar los efectos obtenidos durante un período mayor de intervención, con más meses de ejercicio y aplicarlo en ambos géneros, estratificando los resultados por grupo de edad. Otra recomendación es aumentar el tamaño de muestra, lo que por consecuencia, dará un aumento y mayor poder estadístico inferencial al analizar los resultados del experimento y consecuentemente la posibilidad de extrapolar los resultados obtenidos a sujetos con características similares.

Agradecimientos. La presente investigación contó como fuente de apoyo financiero a la convocatoria 2011 de movilidad académica entre la Asociación Nacional de Universidades e Instituciones de Educación Superior en 
México (ANUIES) y la Secretaria General Consejo Superior Universitario Centroamericano (CSUCA). Y la $16^{\circ}$ convocatoria interna del proyectos de investigación de la Universidad Autónoma de Baja California UABC; 149/2/C/13/16.

\section{Resumen}

Introducción: La disminución de la función del sistema inmune con la edad es asociada a la incidencia de enfermedades infecciosas del tracto respiratorio superior; por otro lado, el ejercicio físico ha sido ampliamente recomendado para la salud. Sin embargo se requiere aún fundamentar con mayor exactitud los efectos del ejercicio físico en el sistema inmunológico en adultos mayores. Objetivo: Evaluar el efecto de un programa de hidrogimnasia sobre la concentración sérica de Inmunoglobulina A (IgA) en adultas mayores. Métodos: 26 participantes fueron asignadas aleatoriamente a un grupo experimental (n: 16) y un grupo control (n: 10). Se determinó la concentración sérica de IgA por nefelometría (BN2 Analyser, DADE Behring). Las sesiones de hidrogimnasia se realizaron cinco veces por semana durante 12 semanas; el ejercicio fue aeróbico a una intensidad de 50-60\% de la frecuencia cardíaca máxima controlada por telemetría (Polar-FT7). Resultados: Se utilizó la prueba de ANOVA, indicando no interacción significativa (p: 0,797$)$. El porcentaje de cambio resultó de $\Delta \%=-6,7 \mathrm{mg} / \mathrm{dL}$ del grupo experimental en comparación con el grupo control $\Delta \%=-7,4$ $\mathrm{mg} / \mathrm{dL}$. Conclusiones: Un programa de tres meses de hidrogimnasia en mujeres no mejora significativamente los niveles de IgA. Sin embargo, los datos refieren una mejora positiva el porcentaje de cambio $\Delta \%$.

\section{Referencias bibliográficas}

1.- Gleeson M, Walsh N P, British Association of S \& Exercise S. The BASES expert statement on exercise, immunity, and infection. J Sports Sci 2012; 30 (3): 321-4.

2.- Fica C A, Hernández C L, Porte T L, Castro S M, Weitzel T. Respiratory infections caused by metapneumovirus in elderly patients. Rev Chilena Infectol 2011; 28 (2): $174-8$

3.- Hakim F T, Gress R E. Immunosenescence: deficits in adaptive immunity in the elderly. Tissue Antigens 2007; 70 (3): 179-89.

4.- Ripoll M E, Valenzuela B M, Vergara F R, Abarca V K, Muñoz M A, Jiménez de la Jara J, et al. 23-valent pneumococcal vaccine. Statement of the Consultive Committee of Immunizations on behalf of the Chilean Infectious Diseases Society: February 2010. Rev Chilena Infectol 2010; 27 (2): 133-7.

5.- Dorshkind K, Montecino-Rodríguez E, Signer R A. The ageing immune system: is it ever too old to become young again? Nat Rev Immunol 2009; 9 (1): 57-62.

6.- Fulop T, Larbi A, Hirokawa K, Mocchegiani E, Lesourds B, Castle S, et al. Immunosupportive therapies in aging. Clin Interv Aging 2007; 2 (1): 33-54.

7.- Neubauer O, Reichhold S, Nersesyan A, Konig D, Wagner K H. Exercise-induced DNA damage: is there a relationship with inflammatory responses? Exerc Immunol Rev 2008; 14: 51-72.

8.- Senchina D S, Kohut M L. Immunological outcomes of exercise in older adults. Clin Interv Aging 2007; 2 (1): 3-16.

9.- Kokkinos P, Myers J, Faselis C, Panagiotakos D B, Doumas M, Pittaras A, et al. Exercise capacity and mortality in older men: a 20-year follow-up study. Circulation 2010; 122 (8): 790-7.

10.- Stamatakis E, Hamer M, Dunstan D W. Screen-based entertainment time, allcause mortality, and cardiovascular events: population-based study with ongoing mortality and hospital events follow-up. J Am Coll Cardiol 2011; 57 (3): 292-9.

11.- Woods J A, Vieira V J, Keylock K T. Exercise, inflammation, and innate immunity. Immunol Allergy Clin North Am 2009; 29 (2): 381-93.

12.- García P, Bahamondes L, Reyes $P$, Román J C, Poblete H, Balcells M E. A comparative study for adenosine deaminase and anti-antigen A-60 antibodies detection for the diagnosis of tuberculous meningitis. Rev Chilena Infectol 2012; 29 (5): 521-6.

13.- Kohut M L, Senchina D S. Reversing ageassociated immunosenescence via exercise. Exerc Immunol Rev 2004; 10: 6-41.

14.- Buyukyazi G, Kutukculer N, Kutlu N, Genel F, Karadeniz G, Ozkutuk N. Differences in the cellular and humoral immune system between middle-aged men with different intensity and duration of physically training. J Sports Med Phys Fitness 2004; 44 (2): 207-14

15.- Shimizu K, Kimura F, Akimoto T, Akama T, Otsuki T, Nishijima T, et al. Effects of exercise, age and gender on salivary secretory immunoglobulin A in elderly individuals. Exerc Immunol Rev 2007; 13: 55-66.

16.- Sakamoto Y, Ueki S, Kasai T, Takato J, Shimanuki H, Honda H, et al. Effect of exercise, aging and functional capacity on acute secretory immunoglobulin A response in elderly people over 75 years of age. Geriatr Gerontol Int 2009; 9 (1): 81-8.

17.- Martins R A, Cunha M R, Neves A P, Martins M, Teixeira-Verissimo M,
Teixeira A M. Effects of aerobic conditioning on salivary IgA and plasma IgA, IgG and IgM in older men and women. Int J Sports Med 2009; 30 (12): 906-12.

18.- Akimoto T, Kumai Y, Akama T, Hayashi E, Murakami H, Soma R, et al. Effects of 12 months of exercise training on salivary secretory IgA levels in elderly subjects. Br J Sports Med 2003; 37 (1): 76-9.

19.- Lim Y M, Hong G R. Effect of 16-week KoukSun-Do exercise on physical fitness, emotional state, and immunoglobulin A in communitydwelling elders in Korea. Appl Nurs Res 2010; 23 (2): 91-100.

20.- Thomas J R, Nelson J K, Silverman S J. Research Methods in Physical Activity 6th. Ed. Champaign, Ilinois: Human Kinetics; 2011: 136-61.

21.- Trujillo A J, Mroz T A, Piras C, Angeles G, Tran N. Caregiving and elderly health in Mexico. Int J Health Serv 2012; 42 (4): 667-94.

22.- Barreto A C, Ribeiro L G. Determinação do tamanho amostral. Fitness \& Perform J 2004; 3 (3): 124.

23.- Puri K S, Suresh K R, Gogtay N J, Thatte U M. Declaration of Helsinki, 2008: implications for stakeholders in research. J Postgrad Med 2009; 55 (2): 131-4.

24.- American College of Sports M, Chodzko-Zajko W J, Proctor D N, Fiatarone Singh M A, Minson C T, Nigg C R, Skinner J S. American College of Sports Medicine position stand. Exercise and physical activity for older adults. Med Sci Sports Exerc 2009; 41 (7): 1510-30.

25.- Nelson M E, Rejeski W J, Blair S N, Duncan P W, Judge J O, King A C, American Heart A. Physical activity and public health in older adults: recommendation from the 
American College of Sports Medicine and the American Heart Association. Circulation 2007; 116 (9): 1094-105.

26.- Tanaka H, Monahan K D, Seals D R. Age-predicted maximal heart rate revisited. J Am Coll Cardiol 2001; 37 (1): 153-6.

27.- Teixeira A M, Martins R A, Martins M, Cunha M R. Changes in functional fitness, mood states and salivary iga levels after exercise training for 19 weeks in elderly subjects. International journal of applied sports sciences 2008; 20 (2): 16-26

28.- Kamioka H, Tsutani K, Mutoh Y, Okuizum H, Ohta M, Handa S, et al. A systematic review of nonrandomized controlled trials on the curative effects of aquatic exercise. Int J Gen
Med 2011; 4: 239-60.

29.- Kamioka H, Tsutani K, Okuizumi H, Mutoh Y, Ohta M, Handa S, et al. Effectiveness of aquatic exercise and balneotherapy: a summary of systematic reviews based on randomized controlled trials of water immersion therapies. J Epidemiol 2010; 20 (1): $2-12$ 